Originalien

Anaesthesist 2020 69:162-169

https://doi.org/10.1007/s00101-020-00736-3

Eingegangen: 16. Oktober 2019

Überarbeitet: 12. Dezember 2019

Angenommen: 14. Januar 2020

Online publiziert: 13 . Februar 2020

(c) Der/die Autor(en) 2020

F. Schneider ${ }^{1,14}$ - C. M. Schulz $\cdot$ M. May ${ }^{2}$ G. Schneider ${ }^{1} \cdot$ M. Jacob J H. Mutlak $^{4}$. M. Pawlik ${ }^{5}$ M. Zoller ${ }^{6}$ M. Kretzschmar . C. Koch ${ }^{8}$ - M. G. Kees ${ }^{9} \cdot$ M. Burger ${ }^{10}$.

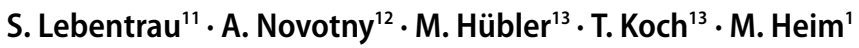

${ }^{1}$ Fakultät für Medizin, Klinik für Anästhesiologie und Intensivmedizin, Technische Universität München, München, Deutschland; ' ${ }^{2}$ Urologische Klinik, St. Elisabeth-Klinikum Straubing, Straubing, Deutschland; ${ }^{3}$ Klinik für Anästhesiologie, Operative Intensivmedizin und Schmerzmedizin, Klinikum St. Elisabeth Straubing, Straubing, Deutschland; ${ }^{4}$ Klinik für Anästhesiologie, Intensivmedizin und Schmerztherapie, Universitätsklinikum Frankfurt, Goethe-Universität, Frankfurt, Deutschland; ${ }^{5}$ Klinik für Anästhesiologie, Krankenhaus St. Josef Regensburg, Regensburg, Deutschland; ${ }^{6}$ Klinik für Anästhesiologie der Universität München, Klinikum der Ludwig-Maximilians-Universität München, München, Deutschland; ${ }^{7}$ Klinik für Anästhesiologie und Intensivtherapie, Universitätsklinikum Magdeburg A.ö.R., Otto-von-GuerickeUniversität Magdeburg, Magdeburg, Deutschland; ${ }^{8} \mathrm{Klinik}$ für Anästhesiologie, operative Intensivmedizin und Schmerztherapie, Justus-Liebig-Universität Gießen, Gießen, Deutschland; ${ }^{9}$ Klinik für Anästhesiologie, Universitätsklinikum Regensburg, Regensburg, Deutschland; ${ }^{10}$ Urologische Klinik, Caritas St. Josef Krankenhaus, Universität Regensburg, Regensburg, Deutschland; " Urologische Klinik, Ruppiner Kliniken GmbH, Medizinische Hochschule Brandenburg Theodor Fontane, Neuruppin, Deutschland; ${ }^{12}$ Fakultät für Medizin, Klinik und Poliklinik für Chirurgie, Technische Universität München, München, Deutschland; ${ }^{13}$ Klinik für Anästhesiologie und Intensivtherapie, Universitätsklinikum Carl Gustav Carus, Technische Universität Dresden, Dresden, Deutschland; ${ }^{14} \mathrm{Klinik}$ für Anästhesiologie und Intensivmedizin, Klinikum rechts der Isar, Technische Universität München, München, Deutschland

\title{
Ist die Fachrichtung assoziiert mit der Selbstsicherheit im Umgang mit rationaler Antibiotikaverordnung?
}

\section{Ergebnisse der MR2-Studien an deutschen Kliniken}

\section{Zusatzmaterial online}

Die Online-Version dieses Beitrags (https:// doi.org/10.1007/s00101-020-00736-3) enthält weitere Tabellen zur detaillierteren Auswertung und die der Studie zugrunde liegenden Fragebogen. Beitrag und Zusatzmaterial stehen Ihnen auf www. springermedizin.de zur Verfügung. Bitte geben Sie dort den Beitragstitel in die Suche ein; das Zusatzmaterial finden Sie beim Beitrag unter „Ergänzende Inhalte“.

\section{Hintergrund}

Neben Fachkenntnis wurde bereits mehrfach Selbstbewusstsein als Schlüsselfaktor für die klinische Entscheidungsfindung beschrieben $[1,2]$. Darüber hinaus beeinflusst das Selbstbewusstsein, synonym zum Vertrauen in die eigenen Fähigkeiten und Urteile, auch die Motiva- tion und den Arbeitserfolg [3, 4]. Interessanterweise werden mit dem Begriff auch eine niedrigere/höhere Zufriedenheit mit der Weiterbildung und eine niedrigere/höhere Hemmschwelle bei der Bitte um Unterstützung in Verbindung gebracht [5]. In einer Studie zum Selbstbewusstsein in Bezug auf palliativmedizinische Fragestellungen konnten Storarri et al. zeigen, dass Studierende der höheren Semester sich deutlich selbstbewusster über dieses Thema äußern als jüngere Kommilitonen [6]. Diese Ergebnisse decken sich mit einer Analyse, die zeigen konnte, dass eine zusätzliche Zertifizierung (Zusatzbezeichnung Palliativmedizin) das Selbstbewusstsein in diesem Bereich steigert [7]. Im Hinblick auf die Verordnung von Antibiotika wurde gezeigt, dass das Vorhandensein der Zusatzbezeichnung Intensivmedizin po- sitiv mit einem diesbezüglichen Selbstbewusstsein assoziiert war [8].

Die unsachgemäße Verordnung von Breitspektrumantibiotika ist ein wichtiger Faktor, der zur rasanten Resistenzausbreitung beiträgt [9]. Die Gründe hierfür sind vielschichtig; in ihrer Übersichtsarbeit zum unangemessenen Antibiotikaeinsatz in Europa nennen Machowska und Lundborg neben dem Wissensmangel in der Bevölkerung auch die problematische Selbstsicherheit von Allgemeinärzten, die die zunehmenden Antibiotikaresistenzen zwar wahrnehmen, sie jedoch nicht als sie selbst betreffendes Problem einordnen [10]. Von infektiologischer Seite wird auch das Fehlen eines designierten Facharzttitels für Infektiologie und der daraus resultierende Mangel an ausreichender konsiliarischer Beratung derklinischen Fachabteilungen als 
weitere Ursache für problematische Antibiotikaverwendung genannt, denn gerade im Kontext von Infektionen mit multiresistenten Erregern können innovative Therapieansätze von entsprechend qualifiziertem Personal zur Steigerung der Therapieeffizienz führen [11]. Eine Infektionsbehandlung ausschließlich durch designierte internistische Infektiologen, wie sie von den entsprechenden Fachgesellschaften diskutiert wird, sollte jedoch kritisch betrachtet werden [12, 13].

Während die Selbstwahrnehmung der Allgemeinärzte in Bezug aufden Antibiotikaeinsatz und die hausärztliche Wahrnehmung von Problemen, bedingt durch die zunehmenden Resistenzen, bereits gut untersucht wurde, ist wenig über die Krankenhausärzte sowie die Charakteristika verschiedener Facharztgruppen bekannt [14].

Im Jahr 2015 wurden 1061 Ärztinnen und Ärzte der Fachrichtungen innere Medizin, Allgemeinchirurgie, Gynäkologie und Urologie mithilfe des Multiinstitutional Reconnaissance of practice with MultiResistant bacteria (MR2) Survey über ihre Einstellung und ihr Wissen zu Antibiotika und multiresistenten Erregern befragt [15-22]. Ein ähnlicher Fragebogen wurde 2017 an 1268 Fach- und Assistenzärzte der Anästhesiologie versendet [15-22]. Ziel der vorliegenden, explorativen Arbeit war die erstmalige gemeinsame Analyse von beiden MR2-Studien, um dadurch potenzielle fachspezifische Unterschiede der Selbstsicherheit im Umgang mit Antibiotika und auch der Selbstwahrnehmung des eigenen Wissens über Multiresistenzen sowie die rationale antimikrobielle Therapie zu untersuchen.

\section{Material und Methoden}

\section{Studiensetting}

Die Daten für die vorliegende Analyse stammen aus 2 Studien. Die Daten zu den Mitarbeitern der Fachabteilungen für innere Medizin, Allgemeinchirurgie, Gynäkologie und Urologie wurden 2015 an 18 deutschen Kliniken erhoben [15-17]. Hierbei wurden 1061 Fragebogen an 6 Universitätskliniken sowie 12 Krankenhäuser der Tertiärversorgung gesendet, jedoch betrieben nicht alle beteiligten Krankenhäuser Abteilungen aller beteiligten Fachrichtungen. Die Daten zur Fachrichtung Anästhesie wurden 2017 in einer MR2-Nachfolgestudie an 16 anästhesiologischen Hauptabteilungen deutscher Krankenhäuser (7 Universitätskliniken, ein Primär-, 6 Sekundär- und 2 Tertiärversorger) erhoben [18-20]. Die Studie wurde bei der Bayerischen Ärztekammer (BLÄK) registriert; ein darüber hinausgehendes Ethikvotum war nicht erforderlich (BLÄK-Registrierungsnummer 18-040).

Beide Fragebogen umfassten neben demografischen Items auch Fragen zur Selbstsicherheit im Umgang mit Antibiotika sowie zur Selbsteinschätzung des eigenen Wissens über die rationale antimikrobielle Therapie. Es waren 4 Fragen zur Selbstsicherheit und 11 Fragen zur Selbsteinschätzung des Wissens in beiden Untersuchungen identisch. Diese bildeten damit die Grundlage der vorliegenden Analyse. Genaue Informationen zur Fragebogenentwicklung sowie Details zur Datenanalyse wurden in vorangegangenen Publikationen hinterlegt $[16,19]$. Der Fragebogen für die chirurgischen, internistischen, gynäkologischen und urologischen Fach- und Assistenzärzte ist im Zusatzmaterial online ESM A dargestellt, der Fragebogen für die Ärzte der Anästhesiologie hingegen im Zusatzmaterial online ESM B.

\section{Strukturierung der Fragebogen}

Beide Fragebogen enthielten neben einer Frage zur Einschätzung der Resistenzrate von Escherichia Coli gegen Ciprofloxacin am eigenen Klinikum 4 gleichlautende Items zum Selbstbewusstsein im Umgang mit der Antibiotikaverordnung. Das Selbstbewusstsein konnte hierbei mittels einer 4-stufigen Likert-Skala mit den Antwortmöglichkeiten: 1: sehr unsicher, 2: unsicher, 3: sicher, 4: sehr sicher bewertet werden. Darüber hinaus enthielten die Fragebogen 11 Items zur Selbsteinschätzung des eigenen Wissens über multiresistente Erreger sowie die rationale Antibiotikaverordnung (ebenfalls 4-stufige Likert-Skala; 1: keine Kenntnisse, 2: geringe Kenntnisse, 3: durchschnittliche Kenntnisse, 4: vollständige Kenntnisse).
Zusätzlich wurden von den Teilnehmern Fachrichtung, Ausbildungsstand, Anzahl der besuchten Fortbildungen zu einem infektiologischen Thema innerhalb des letzten Jahres und die Position in der Klinik erfragt. Die Ansprechpartner der jeweiligen Kliniken wurden gebeten, einige krankenhausinterne Kennzahlen (Resistenzraten von Escherichia Coli gegen Ciprofloxacin) anzugeben.

\section{Statistische Analyse}

Für beide Studiengruppen wurden alle zurückgesendeten Fragebogen zentral begutachtet und auf Plausibilität geprüft $[16,19]$. In Übereinstimmung mit den vorangegangenen Veröffentlichungen zum Thema wurden die Werte der Likert-Skalen zur besseren Darstellbarkeit von Unterschieden zwischen den Gruppen als Mittelwerte und Standardabweichung angegeben [16, 18-21]. Für die explorative statistische Auswertung wurden nominale Variablen mithilfe des Chi-Quadrat-Tests verglichen, während der Kruskal-Wallis-Test für ordinale Variablen gewählt wurde. Zusätzlich wurde der Einfluss der Fachrichtung auf die Studienkriterien mittels eines logistischen Regressionsmodelles (LRM) untersucht: Hierbei wurden die Antworten auf die Items dichotomisiert (sehr unsicher und unsicher $v s$. sicher und sehr sicher; keine Kenntnisse und geringe Kenntnisse vs. durchschnittliche Kenntnisse und vollständige Kenntnisse). Zusätzlich zu der Fachrichtung des Teilnehmers wurden 1) die Erfahrung des Arztes (Assistenzarzt vs. Facharzt), 2) seine Position in der Klinik (Oberarzt/Chefarzt vs. Facharzt/Assistenzarzt) und 3) die Anzahl der spezifischen Fortbildungen, die während der letzten 12 Monate besucht wurden, in das Modell eingeschlossen.

Ein 2-seitiges Signifikanzniveau von $p<0,05$ wurde als signifikant betrachtet und SPSS Statistics 24.0 (IBM Corp., Armonk, NY, USA) zur Datenanalyse eingesetzt. 
Anaesthesist 2020 • 69:162-169 https://doi.org/10.1007/s00101-020-00736-3

(c) Der/die Autor(en) 2020

F. Schneider · C. M. Schulz · M. May · G. Schneider · M. Jacob · H. Mutlak · M. Pawlik · M. Zoller · M. Kretzschmar · C. Koch · M. G. Kees · M. Burger · S. Lebentrau · A. Novotny · M. Hübler · T. Koch · M. Heim

\section{Ist die Fachrichtung assoziiert mit der Selbstsicherheit im Umgang mit rationaler Antibiotikaverordnung? Ergebnisse der MR2-Studien an deutschen Kliniken}

\section{Zusammenfassung}

Hintergrund. Vor dem Hintergrund einer stetig zunehmenden Gesundheitsgefährdung durch multiresistente Erreger spielt neben der Bevölkerungsaufklärung, der Fachkenntnis und der Fortbildung der Ärztinnen und Ärzte auch deren Selbstsicherheit eine Schlüsselrolle in der klinischen Entscheidungsfindung. Ziel der Arbeit. Die Analyse soll den Einfluss der Fachrichtung auf Unterschiede der Selbstsicherheit im Umgang mit Antibiotika und in der Selbsteinschätzung des eigenen Wissens untersuchen.

Methodik. Im Jahr 2015 wurden 1061 Ärztinnen und Ärzte der Fachrichtungen Innere Medizin, Allgemeinchirurgie, Gynäkologie und Urologie mithilfe des MR2 (Multiinstitutional Reconnaissance of practice with MultiResistant bacteria)-Surveys über ihre Einstellung und ihr Wissen zu Antibiotika und multiresistenten Erregern befragt. Ein ähnlicher Fragebogen wurde 2017 an 1268 Ärzte der Anästhesiologie versendet. Es wurden vier Fragen zur eigenen Sicherheit in der Anwendung von Antibiotika und elf Fragen zur Einschätzung des eigenen Wissens über multiresistente Erreger und rationale Antibiotikatherapie ausgewertet. Der Einfluss der Fachrichtung auf diese Items wurde mittels einer logistischen Regressionsanalyse, eines "chi square tests" und des KruskalWallis-Tests untersucht.

Ergebnisse. Die Rücklaufquoten betrugen $43 \%(456 / 1061)$ und $56 \%$ (705/1268). Im Jahr vor der Umfrage haben $44 \%$ der NichtAnästhesisten und $57 \%$ der Anästhesisten keine Fortbildung zu Inhalten des Antibiotic Stewardships besucht. Im Hinblick auf die Selbstsicherheit im Umgang mit Antibiotika zeigten sich Anästhesisten (Mittelwert \pm Standardabweichung: 2,53 $\pm 0,54$ ) signifikant unsicherer als ihre Kollegen (Innere Medizin:

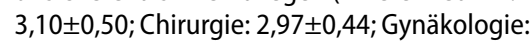
$3,12 \pm 0,42$; Urologie: $3,15 \pm 0,44$ ); dies gilt sowohl für die nicht-adjustierten (alle $p<0,001)$, als auch die adjustierten Vergleiche. Ähnliche Ergebnisse zeigten sich für die Selbsteinschätzung des eigenen Wissens. Facharztstatus und der Besuch von infektiologischen Fortbildungen waren signifikant mit einem besseren Selbstbewusstsein als auch einer höheren Selbsteinschätzung des eigenen Wissens assoziiert.

Schlussfolgerungen. Anästhesisten zeigten eine signifikant geringere Selbstsicherheit im Umgang mit Antibiotika als ihre Kollegen aus anderen Fachrichtungen. Fortbildungen zur rationalen Verordnung von Antibiotika waren mit einer höheren Handlungssicherheit assoziiert, so dass eine Verankerung in den jeweiligen Weiterbildungsinhalten zu diskutieren ist.

Schlüsselwörter

Antibiotika - Entscheidungsfindung · Facharztweiterbildung · Mikrobiologische Diagnostik • „Antibiotic stewardship“

\section{Is the discipline associated with self-confidence in handling rational antibiotic prescription? Results from the MR2 study in German hospitals}

\section{Abstract}

Background. Besides public awareness and specialist knowledge and training of physicians, their self-confidence plays a key role for clinical decision-making in the respective area.

Objective. This exploratory study investigated the influence of the discipline on differences in self-confidence in dealing with antibiotics and in the self-rated knowledge.

Methods. In 2015 the multi-institutional reconnaissance of practice with multiresistant bacteria (MR2) questionnaire containing items on antibiotic prescription and multiresistant pathogens was sent out to 1061 physicians working in departments for internal medicine, general surgery, gynecology and obstetrics and urology. In 2017 a similar MR2 survey was sent to 1268 specialist and assistant physicians in anesthesiology in Germany. Besides demographic data 4 items on self-confidence in the use of antibiotic treatment and 11 items concerning self-rated knowledge about rational antibiotic therapy and multiresistant pathogens were included in the present analysis. Logistic regression analysis, the $x^{2}-$ test and the Kruskal-Wallis test were used for statistical analysis of the influence of the discipline on these items.

Results. The response rates were $43 \%$ (456 out of 1061) from the non-anesthetists and $56 \%$ (705 out of 1268) from the anesthetists. Of the non-anesthetists $44 \%$ and $57 \%$ of the anesthetists had had no advanced training on antibiotic stewardship during the year before the study. In the overall analysis anesthetists (mean \pm SD: $2.53 \pm 0.54$ ) were significantly less self-confident about antibiotics than colleagues from other departments (internal medicine: $3.10 \pm 0.50$, general surgery: $2.97 \pm 0.44$, gynecology and obstetrics: $3.12 \pm 0.42$ and urology: $3.15 \pm 0.44$ ) in the unadjusted (all $p<0.001$ ) and adjusted comparison. The analysis of selfrated knowledge about rational antibiotic prescription showed similar results. Senior consultant status and advanced training in infectiology were significantly associated with self-confidence and self-rated knowledge about antibiotics.

Conclusion. Anesthetists showed significantly less self-confidence in dealing with antibiotics than colleagues from other disciplines. Advanced training on a rational prescription of antibiotics was associated with a greater self-confidence, so that the implementation of compulsory courses on rational antibiotic stewardship in the respective residency curriculum needs to be considered.

Keywords

Antibiotics - Clinical decision making · Specialization - Microbiological diagnostics . Antimicrobial stewardship 


\begin{tabular}{|c|c|c|c|c|c|}
\hline & $\begin{array}{l}\text { Innere Medi- } \\
\text { zin }\end{array}$ & Chirurgie & Gynäkologie & Urologie & Anästhesie \\
\hline \multicolumn{6}{|c|}{ Position in der Klinik } \\
\hline $\begin{array}{l}\text { Chef-/ } \\
\text { Oberarzt }\end{array}$ & $58(43,9 \%)$ & $65(41,7 \%)$ & $10(30,3 \%)$ & $56(41,5 \%)$ & $156(23,1 \%)$ \\
\hline Facharzt & $18(13,6 \%)$ & $31(19,9 \%)$ & $13(39,4 \%)$ & $28(20,7 \%)$ & $205(30,3 \%)$ \\
\hline Assistenzarzt & $56(42,2 \%)$ & $60(38,5 \%)$ & $10(30,3 \%)$ & $51(37,8 \%)$ & $315(48,6 \%)$ \\
\hline \multicolumn{6}{|c|}{ Patientenzahl mit eigenständiger Verabreichung eines Antibiotikums in den letzten 7 Tagen } \\
\hline Kein Patient & $18(13,7 \%)$ & $17(11,0 \%)$ & $6(19,4 \%)$ & $8(6,0 \%)$ & $164(24,3 \%)$ \\
\hline 1-2 Patienten & 45 (34,4\%) & $52(33,5 \%)$ & $9(29,0 \%)$ & $25(18,7 \%)$ & $107(15,8 \%)$ \\
\hline 3-5 Patienten & $41(31,3 \%)$ & $40(25,8 \%)$ & $12(38,7 \%)$ & $33(24,6 \%)$ & $109(16,1 \%)$ \\
\hline >5 Patienten & $27(20,6 \%)$ & $46(29,7 \%)$ & $4(12,9 \%)$ & $68(50,7 \%)$ & $296(43,8 \%)$ \\
\hline \multicolumn{6}{|c|}{$\begin{array}{l}\text { Anzahl der besuchten Fortbildungsveranstaltungen zum Thema Antibiotika in den letzten } \\
12 \text { Monaten }\end{array}$} \\
\hline Keine & $62(49,2 \%)$ & $66(44,6 \%)$ & $14(43,8 \%)$ & $47(37,0 \%)$ & $384(57,1 \%)$ \\
\hline Eine & $47(37,3 \%)$ & $67(45,3 \%)$ & $15(46,9 \%)$ & $58(45,7 \%)$ & $239(35,5 \%)$ \\
\hline Mehr als eine & $17(13,5 \%)$ & $15(10,1 \%)$ & $3(9,4 \%)$ & $22(17,3 \%)$ & $50(7,4 \%)$ \\
\hline \multicolumn{6}{|c|}{ Ciprofloxacinresistenzrate von Escherichia Coli in der eigenen Abteilung ${ }^{a}$} \\
\hline Unterschätzt & $62(47,0 \%)$ & $100(64,1 \%)$ & $21(63,6 \%)$ & $59(43,7 \%)$ & $298(43,6 \%)$ \\
\hline Überschätzt & $22(16,7 \%)$ & $13(8,3 \%)$ & $3(9,1 \%)$ & $27(20,0 \%)$ & $61(8,9 \%)$ \\
\hline Korrekt & $48(36,4 \%)$ & $43(27,6 \%)$ & $9(27,3 \%)$ & $49(36,3 \%)$ & $141(20,6 \%)$ \\
\hline $\begin{array}{l}\text { Keine } \\
\text { Angabe }^{b}\end{array}$ & $0(0,0 \%)$ & $0(0,0 \%)$ & $0(0,0 \%)$ & $0(0,0 \%)$ & $184(26,9 \%)$ \\
\hline \multicolumn{6}{|c|}{$\begin{array}{l}\text { - Tab. } 1 \text { zeigt die deskriptiven Informationen zur Studiengruppe. Die Daten werden als Anzahl } \\
\text { sowie Häufigkeit innerhalb der Gruppe dargestellt } \\
\text { "Krankenhausbezogene Daten, die auf die einzelnen Teilnehmer verteilt wurden } \\
\text { 'Die Kategorie „keine Angabe" war nur im Fragebogen des anästhesiologischen Studienzweigs } \\
\text { enthalten }\end{array}$} \\
\hline
\end{tabular}

\section{Ergebnisse}

\section{Rücklaufquote}

Im zuerst durchgeführten (interdisziplinären) Studienarm betrug die Gesamtrücklaufquote $43 \%$ (456/1061). Die separaten Rücklaufquoten waren hierbei: 29,1\% (132/454; innere Medizin), 47,3\% (156/330; Allgemeinchirurgie), $32,7 \%$ (33/101; Gynäkologie) und 76,7 \% (135/176; Urologie). Im anästhesiologischen Studienarm betrug die Rücklaufquote 55,6\% (705/1268). Die erhobenen demografischen Daten der Studiengruppe werden in $\bullet$ Tab. 1 dargestellt.

\section{Unterschiede in der Selbst- sicherheit bei der Antibiotika- verwendung zwischen den Fachrichtungen}

Im Umgang mit Antibiotika fühlten sich Fach- und Assistenzärzte der Anästhesiologie im Mittel weniger selbstsicher als keine Fortbildung zu Inhalten des „antibiotic stewardship“ (ABS). Unabhängig von ihrer Fachrichtung fühlten sich die Teilnehmer, die an mindestens einer Fortbildung teilgenommen hatten, sowohl im Mittelwertvergleich $(p<0,001)$ als auch nach Adjustierung um mögliche Einflussfaktoren $(p=0,003)$ selbstsicherer als jene Kollegen, die keine Fortbildung besucht hatten. Zusätzlich schätzten sie auch ihr Wissen über den rationalen Einsatz von Antibiotika deutlich besser ein $(p<0,001)$. Sowohl der Facharztstatus als auch die Positionen Oberarzt bzw. Chefarzt (Klinikdirektor) waren im Mittelwertvergleich mit einem einen positiven Einfluss auf das Selbstbewusstsein assoziiert. Dieses Ergebnis hatte im LRM jedoch nur für den Facharztstatus Bestand. Details zu den $p$-Werten sowie die zugehörigen Mittelwerte finden sich in - Tab. 3. Das Zusatzmaterial online ESM D gibt eine Übersicht über die zusätzliche Auswertung einzelner Fragen.

\section{Diskussion}

Ziel der vorliegenden, explorativen Studie war es, die Assoziation der medizinischen Fachrichtung mit der Selbstsicherheit im Umgang mit Antibiotika sowie die Selbstwahrnehmung des eigenen Wissens über Multiresistenzen und die rationale antimikrobielle Therapie zu untersuchen. Bezüglich der im MR2-Fragebogen abgefragten Themenbereiche fühlten sich Anästhesisten deutlich unsicherer im Umgang mit Antibiotika als die Kollegen der anderen betrachteten Fachrichtungen (alle $p<0,001$ ). Ähnliche Ergebnisse zeigten sich im Mittelwertvergleich für die Selbsteinschätzung des eigenen Wissens; hier war jedoch der Einfluss der einzelnen Fachrichtungen im LRM geringer. Besonders der Facharztstatus und der Besuch von infektiologischen Fortbildungen beeinflussten die Selbstsicherheit der Teilnehmer positiv ( $p<0,001$ für Mittelwertvergleich). Basierend auf der vorliegenden Analyse können keine Aussagen über Wissensunterschiede zwischen den verschiedenen Fachdisziplinen getroffen werden.

Für das Ergebnis, dass Fach- und Assistenzärzte der Anästhesiologie bei den verwendeten Fragen eine geringere 


\section{Originalien}
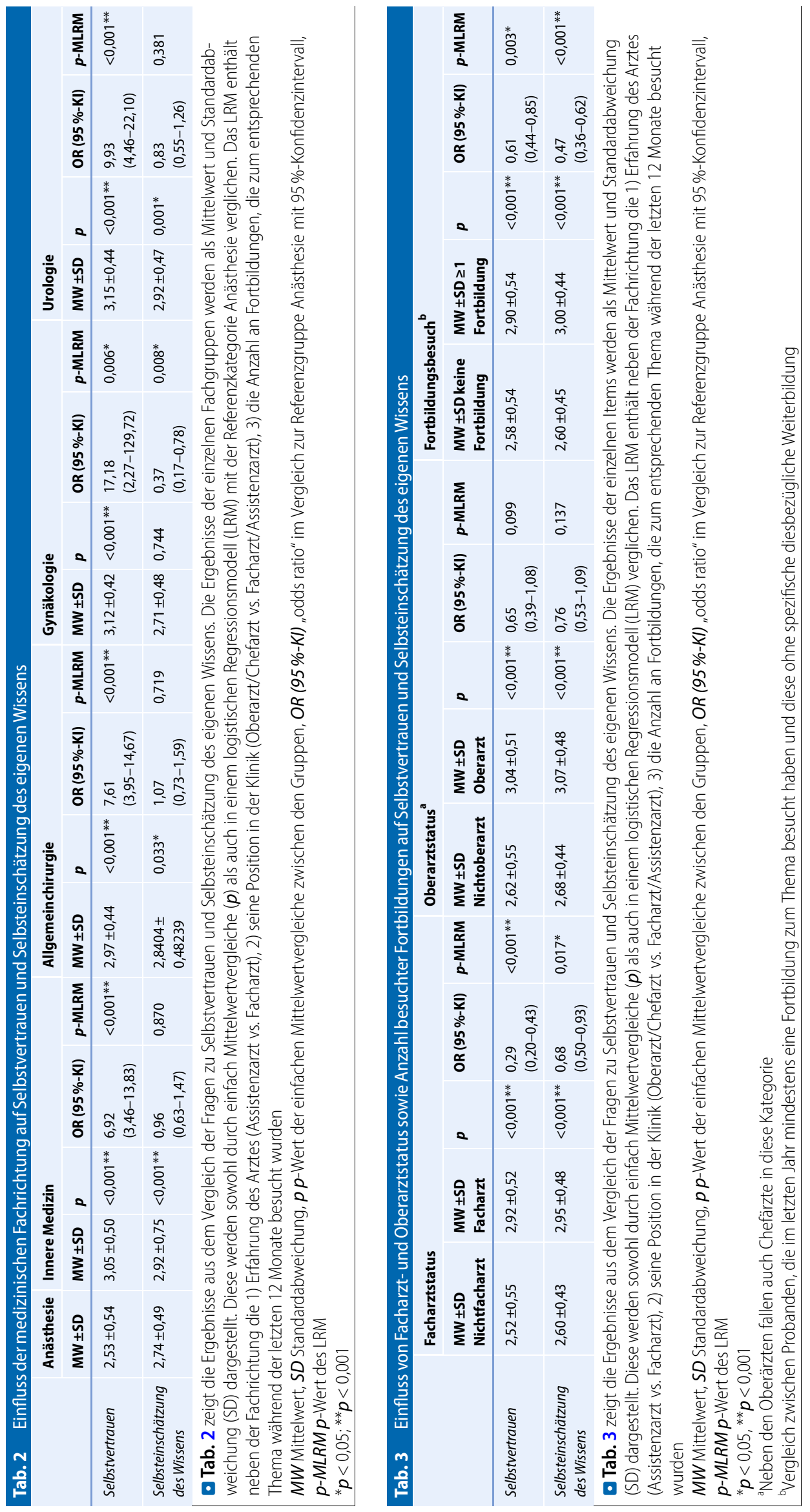
Selbstsicherheit im Umgang mit Antibiotika im Vergleich zu ärztlichen Kollegen anderer Fachabteilungen zeigen, gibt es mehrere mögliche Erklärungen. Da Anästhesisten einerseits häufig in verschiedenen operativen Bereichen eingesetzt werden, in denen die Antibiotikaprophylaxe bzw. -therapie eingriffsspezifisch standardisiert ist, erfolgt in der Regel keine eigenständige Wahl des Antibiotikums. Andererseits sind Anästhesisten bereits während der Weiterbildung mindestens ein Jahr auf der Intensivstation tätig und dort mit der Therapie von kritisch kranken Patienten und dem Umgang mit multiresistenten Erregern konfrontiert. Die mangelnde Erfahrung der im OP tätigen Anästhesisten mit der eigenständigen Auswahl von Antibiotika sowie die anspruchsvolle antimikrobielle Therapie bei komplexen Intensivpatienten könnten die möglichen Ursachen für eine weniger selbstsichere Haltung bei dem zielgerichteten, eigenständigen Einsatz von Antibiotika sein. Dies bedeutet aber keineswegs, dass eine geringere Selbstsicherheit auch mit einem geringeren objektiven Wissen assoziiert ist. In diesem Licht ist die geringere Selbstsicherheit sogar positiv zu bewerten, da sie, vom rein klinischen Hintergrund gelöst, auch eine größere Fähigkeit zur Selbstkritik impliziert und zu Reevaluation und Diskussion einlädt. So kann die antimikrobielle Therapie ggf. durch die Zuhilfenahme externer Expertise (z.B. durch infektiologische Konsile) sowohl gezielt für den Patienten optimiert als auch an die lokalen Erregerspektren und Risiken angepasst werden. Allerdings muss bei der Interpretation des Studienergebnisses auch Berücksichtigung finden, dass Fach- und Assistenzärzte der Anästhesiologie mit einem Mittelwert von 2,53 im Schnitt zwar unsicherer als ihre Kollegen aus den anderen Fachabteilungen sind, sich alle Ärzte aber dennoch mit Mittelwerten zwischen 2,53 und 3,15 auf der vorliegenden Likert-Skala insgesamt eher selbstsicher im Umgang mit Antibiotika fühlen (Interpretation: s. Abschn. „Material und Methoden"). Bisher wurden von verschiedenen Autorengruppen ähnliche Skalen verwendet, um die Selbstsicherheit und das Selbstvertrauen in Bezug auf medizinische
Themenkomplexe zu erfassen $[4,7,22$, 23]. Für stark verwandte Themen wie etwa die Selbsteinschätzung des infektiologischen Wissens von Krankenschwestern [22] oder die Selbstsicherheit bei palliativmedizinischen Fragestellungen im intensivmedizinischen Umfeld [7] waren diese ähnlich gering, während Geoffrion et al. deutlich größere Unterschiede in der Selbstwahrnehmung von gynäkologischen Assistenzärzten vor und nach einer Intervention zur vaginalen Hysterektomie fanden [4]. Dennoch sind die von der vorliegenden Untersuchung nachgewiesenen Unterschiede in der Selbstsicherheit verschiedener Fachdisziplinen als gering einzustufen.

Ähnliche Ergebnisse finden sich in Bezug auf die Selbsteinschätzung des eigenen Wissens im Umgang mit Antibioti$\mathrm{ka}$. Interessanterweise verabreichten die Anästhesisten im vorliegenden Datensatz mit 43,8 \% innerhalb der Gruppe zwar am häufigsten eigenständig Antibiotika bei mehr als 5 Patienten/Woche (•Tab. 1), fühlten sich dabei jedoch am unsichersten (•Tab. 2) und besuchten gleichzeitig seltener themenspezifische Fortbildungen (-Tab. 1). Insofern kann die vorliegende Studie nicht abschließend klären, ob die Ergebnisse durch berechtigte (ehrliche) Unsicherheit der Anästhesisten im Kontext einer komplexen Thematik oder durch unberechtigte Sicherheit der Mitarbeiter anderer Fachabteilungen begründet sind. Da unterschiedliche Selbstsicherheiten nicht gleichbedeutend mit Unterschieden des objektiven Wissens der Teilnehmer sind, hätte der Vergleich der Fachkenntnis, also des objektiven Wissens, zur Klärung einen wesentlichen Beitrag leisten können, war in dieser Arbeit aber leider nicht möglich. Zudem würde die Frage nach einer angemessenen Fachkenntnis selbst in prospektiven Studien kaum zu beantworten sein, da in den operativen Fächern auch infektiologisch die Schwerpunkte in den jeweiligen Fachgebieten liegen, während die Anästhesiologie in der intrahospitalen Patientenversorgung die interdisziplinäre Schnittstelle zwischen allen operativen und nichtoperativen Fächern darstellt.

Eine Übersichtsarbeit von Machowska et al. aus dem Jahr 2018 weist auch auf eine inadäquate Erwartungshaltung und unzureichendes Wissen der Bevölkerung als Ursachen für Fehlnutzung und Fehlverschreibung von Antibiotika hin [10]. Darüber hinaus identifizieren die Autoren allerdings auch die Furcht der behandelnden Ärzte vor Komplikationen sowie deren mangelnde ABS-Erfahrung als Probleme im Umgang mit antimikrobiellen Substanzen [10, 24]. In diesem Kontext wurde von der Weltgesundheitsorganisation (WHO) wiederholt auf die Bedeutung einer angemessenen studentischen Ausbildung zu Antibiotika und deren Verschreibung hingewiesen [25]. Im Einklang mit diesen Empfehlungen stehen die Ergebnisse des General Medical Council in Großbritannien: 10\% der jungen Ärzte trauen sich die Verschreibung von Antibiotika nicht zu, obwohl sie täglich damit konfrontiert werden [26]. Gleichwohl zeigt die vorliegende Analyse, dass nur von einem sehr kleinen Anteil der Ärzte aller Fachrichtungen 2 oder mehr Fortbildungen zum Thema besucht wurden, obgleich sie regelmäßig selbstständig Antibiotika verordneten (• Tab. 1).

Wenig überraschend ist der Besuch von Fortbildungen zum Thema Multiresistenzen und rationale Antibiotikaverwendung mit höherer Selbstsicherheit sowie einer signifikant höheren Einschätzung des eigenen Wissens assoziiert (• Tab. 3). Zu ähnlichen Ergebnissen kam eine Untersuchung von Phillips et al. [26]. Sie fanden in ihrer Studie zum Selbstvertrauen von jungen Assistenzärzten im Umgang mit Vancomycin nach einer Fortbildung zwar ein vermindertes Selbstvertrauen in Bezug auf das initiale Monitoring, andere Unterpunkte des Selbstvertrauens konnten jedoch durch diese Maßnahme gestärkt werden $[25,26,26,27]$. In einer Analyse des Selbstvertrauens von Krankenschwestern im Umgang mit antimikrobiellen Substanzen konnte hingegen kein Unterschied zwischen den Werten vor und nach einer Fortbildung zum Thema ABS gefunden werden [27]. Ferner deuten die Ergebnisse eines Cochrane-Übersichtsartikels darauf hin, dass Interventionen und Fortbildungen eine effektive Maßnahme sein können, um einen leitlinien- 
gerechten Umgang mit antimikrobiellen Substanzen zu erreichen [28].

In ihrer Untersuchung zum Management von Fieberkrämpfen konnten Bashiri et al. zeigen, dass es zwar große Unterschiede im Fachwissen der an der Versorgung beteiligten Ärztegruppen gibt, eine Subspezialisierung einzelner Facharztgruppen dies jedoch nicht weiter beeinflusst [29]. Andere Studien untersuchten das Wissen bezüglich der transkraniellen Magnetstimulation und kamen zu ähnlichen Ergebnissen [30]. Im Gegensatz zu diesen Ergebnissen konnten im anästhesiologischen Studienzweig der MR2-Studie starke Unterschiede zwischen Anästhesisten ohne und mit Zusatzweiterbildung Intensivmedizin gefunden werden [8]. In der gegenwärtigen Analyse sind Facharztund Oberarztstatus durch alle Fachgruppen hindurch mit einer größeren Selbstsicherheit assoziiert; diese Assoziation fällt in der Auswertung der Items zur Selbsteinschätzung des eigenen Wissens geringer aus (- Tab. 3).

Eine andere Arbeit der MR2-Studie zeigte, dass weibliche Anästhesisten zu deutlich geringerem Selbstbewusstsein als ihre männlichen Kollegen im Umgang mit Antibiotika neigen, wenngleich das objektive Wissen in beiden Gruppen gleich war [18]. Ähnliches gilt für Ärzte an Universitätskliniken im Vergleich mit ihren nichtuniversitär tätigen Kollegen [20]. Im Vergleich zwischen Fachärzten und Assistenzärzten der Anästhesie waren Letztere unsicherer und wiesen ein geringeres objektives Wissen auf [19].

Bei der Interpretation der Studienergebnisse sind einige Limitationen $\mathrm{zu}$ beachten: Zunächst handelt es sich bei der Analyse um eine gepoolte Analyse von Fragebogen, die zu verschiedenen Zeitpunkten und mit lediglich ähnlichem, jedoch nicht gleichem Inhalt versendet wurden (Vergleich von Zusatzmaterial online ESM A und B). Einflüsse durch das unterschiedliche Design und Unterschiede in der Länge der Fragebogen auf die Antworten der Studienteilnehmer können nicht ausgeschlossen werden. Darüber hinaus ist die Auswahl der Fachabteilungen nicht repräsentativ, d.h., es wurden nicht alle Fachrichtungen und deren Selbstwahrnehmung zur
Antibiotikaanwendung in die Befragung eingeschlossen, was die Generalisierbarkeit der Studienergebnisse einschränkt. Zudem können die gängigen Probleme in der Auswertung von Fragebogenstudien („social desirability response set“, „non-response bias“) auch für die vorliegende Studie bestehen.

In der Zusammenschau der Ergebnisse aus den MR2-Studien stellten sich die Unterschiede zwischen einzelnen medizinischen Fachrichtungen deutlicher dar als die Unterschiede innerhalb der Fachabteilungen zwischen Ärzten in verschiedenen akademischen und organisatorischen Positionen. Dies unterstreicht abermals die Bedeutung von Spezialisierungen im Bereich ABS sowie die Wichtigkeit des interdisziplinären Monitorings von Antibiotikaverbrauch und -anwendung. Ärzte, die Fortbildungen zum Thema besucht haben, fühlten sich sicherer. Die Wichtigkeit einer kontinuierlichen Fort- und Weiterbildung zu Multiresistenzen und Antibiotikaanwendung scheint folglich evident und selbstverständlich. Die Ergebnisse zeigten allerdings auch, dass nur ein unzureichender Anteil der Ärzte solche Fortbildungen besucht; aus dieser Sicht muss eine Verankerung von ABS in den jeweiligen Weiterbildungsinhalten diskutiert werden.

\section{Fazit für die Praxis}

- Die Ergebnisse dieser Fragebogenstudie zeigen fachübergreifend eine deutliche Unsicherheit bei der rationalen Verordnung von Antibiotika.

- Diesem Problem sollte durch obligat verankerte themenspezifische Fortbildungsangebote bzw. durch eine stärkere Wichtung von „Antibioticstewardship"(ABS)-Inhalten in der Weiterbildungsordnung Rechnung getragen werden.

- Die höhere Unsicherheit der Anästhesisten im Vergleich zu den anderen untersuchten Fachrichtungen kann durchaus in deren Wissen über Komplexität und Bedeutung von ABSMaßnahmen begründet sein.

\section{Korrespondenzadresse

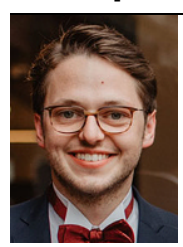 \\ F. Schneider \\ Klinik für Anästhesiologie und Intensivmedizin, Klinikum rechts der Isar, Technische Universität München \\ Ismaninger Str. 22, 81675 München, Deutschland frederick.schneider@tum.de}

Danksagung. Wir möchten uns bei allen Kollegen aus den teilnehmenden Krankenhäusern für Ihre Unterstützung bedanken.

Funding. Open Access funding provided by Projekt DEAL.

\section{Einhaltung ethischer Richtlinien}

Interessenkonflikt. F. Schneider, C.M. Schulz, M. May, G. Schneider, M. Jacob, H. Mutlak, M. Pawlik, M. Zoller, M. Kretzschmar, C. Koch, M.G. Kees, M. Burger, S. Lebentrau, A. Novotny, M. Hübler, T. Koch und M. Heim geben an, dass kein Interessenkonflikt besteht.

Für diesen Beitrag wurden von den Autoren keine Studien an Menschen oder Tieren durchgeführt. Für die aufgeführten Studien gelten die jeweils dort angegebenen ethischen Richtlinien.

Open Access. Dieser Artikel wird unter der Creative Commons Namensnennung 4.0 International Lizenz veröffentlicht, welche die Nutzung, Vervielfältigung, Bearbeitung, Verbreitung und Wiedergabe in jeglichem Medium und Format erlaubt, sofern Sie den/die ursprünglichen Autor(en) und die Quelle ordnungsgemäß nennen, einen Link zur Creative Commons Lizenz beifügen und angeben, ob Änderungen vorgenommen wurden.

Die in diesem Artikel enthaltenen Bilder und sonstiges Drittmaterial unterliegen ebenfalls der genannten Creative Commons Lizenz, sofern sich aus der Abbildungslegende nichts anderes ergibt. Sofern das betreffende Material nicht unter der genannten Creative Commons Lizenz steht und die betreffende Handlung nicht nach gesetzlichen Vorschriften erlaubt ist, ist für die oben aufgeführten Weiterverwendungen des Materials die Einwilligung des jeweiligen Rechteinhabers einzuholen.

Weitere Details zur Lizenz entnehmen Sie bitte der Lizenzinformation auf http://creativecommons.org/ licenses/by/4.0/deed.de.

\section{Literatur}

1. Chuang S-C et al (2013) The impact of selfconfidence on the compromise effect. Int J Psychol 48(4):660-675

2. Fry M, MacGregor C (2014) Confidence and impact on clinical decision-making and behaviour in the emergency department. Australas Emerg Nurs J 17(3):91-97

3. Cervone D (2000) Thinking about self-efficacy. Behav Modif 24(1):30-56 
4. Geoffrion R, Lee T, Singer J (2013) Validating a selfconfidence scale for surgical trainees. J Obstet Gynaecol Can 35(4):355-361

5. Bucholz EM et al (2011) Our trainees' confidence: results from a national survey of 4136 US general surgery residents. Arch Surg 146(8):907-914

6. Storarri ACM et al (2019) Confidence in palliative care issues by medical students and internal medicine residents. BMJ Support Palliat Care 9(1):e1

7. Krautheim V et al (2017) Self-confidence and knowledge of German ICU physicians in palliative care-a multicentre prospective study. BMCPalliat Care 16(1):57

8. Schneider $F$ et al (2020) The association of selfconfidence about rational antibiotic application with academic and educational status: results from the MR2 study. Under review.

9. Richter DC et al (2019) Infections due to multidrug resistant pathogens : pathogens, resistance mechanisms and established treatment options. Anaesthesist 68(10):711-730. https://doi.org/10. 1007/s00101-019-00645-0

10. Machowska A, Stålsby Lundborg C (2018) Drivers of irrational use of antibiotics in Europe. Int J Environ Res Public Health 16(1):E27. https://doi.org/10. 3390/ijerph16010027

11. Richter DC et al (2019) New antibiotics for severe infections due to multidrug-resistant pathogens : definitive treatment and escalation. Anaesthesist 68(11):785-800. https://doi.org/10.1007/s00101019-00646-z

12. Walger $P$ et al (2017) Infektionsmedizin: Klinische Expertise fördern. Dtsch Arztebl 114(19):948

13. Fätkenheuer $G$ et al (2018) Infektiologie - ein Schwerpunkt der Inneren Medizin. Internist 59(4):334-340

14. McCullough AR et al (2015) Not in my backyard: a systematic review of clinicians' knowledge and beliefs about antibiotic resistance. J Antimicrob Chemother 70(9):2465-2473

15. Lebentrau S et al (2017) Impact of the medical specialty on knowledge regarding multidrug-resistant organisms and strategies toward antimicrobial stewardship. Int Urol Nephrol 49(8):1311-1318

16. May $M$ et al (2017) Fühlen sich Chirurgen gerüstet für die komplexen Fragestellungen im Umgang mit multiresistenten Erregern? Ergebnisse der Fragebogenstudie MR2. Zentralbl Chir 142(03):297-305

17. May M et al (2017) Wie werden der 10-PunktePlan des Deutschen Bundesministeriums für Gesundheit zur Bekämpfung resistenter Erreger und Maßnahmen des Antibiotic Stewardship wahrgenommen? Urologe 56(10):1302-1310

18. Heim M et al (2019) Rational application of antibiotics - the influence of anaesthetists' gender on self-confidence and knowledge. Acta Anaesthesiol Scand 63(8):1037-1047

19. May Met al (2019) Kenntnisse und Selbsteinschätzung der Fähigkeiten von anästhesiologischen Ärztinnen und Ärzten in Weiterbildung zu Inhalten einer rationalen Antibiotikaverordnung Ergebnisse einer umfangreichen infektiologischen Fragebogenstudie an deutschen Kliniken. Anasth Intensivmed 60:150-163

20. Schneider F et al (2019) The influence of hospital characteristics on anaesthetists' self-confidence and knowledge about rational antibiotic application-a survey study comparing university and non-university hospitals. Anasth Intensivmed 60(10):468-478

21. Spachmann PJ et al (2019) Awareness and perception of multidrug-resistant organisms and antimicrobial therapy among internists vs. surgeons of different specialties: results from the German MR2 survey. Caspian J Intern Med 10(2):132-141

22. Hale LSetal (2017) Nurses' knowledge, perception, and self-confidence regarding evidence-based antibiotic use in the long-term care setting. Consult Pharm 32(11):700-706

23. Wingen $S$ et al (2018) Self-confidence and level of knowledge after cardiopulmonary resuscitation training in 14 to 18 -year-old schoolchildren: a randomised-interventional controlled study in secondary schools in Germany. Eur J Anaesthesiol 35(7):519-526

24. Gonzalez-Gonzalez C et al (2015) Effect of physicians' attitudes and knowledge on the quality of antibiotic prescription: a cohort study. PLoS One 10(10):e141820

25. Molstad S et al (2017) Lessons learnt during 20 years of the Swedish strategic programme against antibiotic resistance. Bull World Health Organ 95(11):764-773

26. General-Medical-Council (2014) Be prepared: are new doctors safe to practise? https://www. gmc-uk.org/about/what-we-do-and-why/dataand-research/research-and-insight-archive/ how-prepared-are-uk-medical-graduates-forpractice-2014.Zugegriffen:6. Sept. 2019

27. Phillips CJetal (2017) Junior doctors' preparednes to prescribe, monitor, and treat patients with the antibiotic vancomycin in an Australian teaching hospital.JEduc Eval Health Prof 14:13

28. Davey P, Marwick CA, Scott CL et al (2017) Interventions to improve antibiotic prescribing practices for hospital inpatients. The Cochrane database of systematic reviews 2:Cd003543

29. Bashiri FA, Al Shalawi AA, Hamad $M H$ et al (2018) Assessment of physicians' knowledge and attitudes in the management of febrile seizures. Neurosciences 23(4):314-319

30. Alhadi AN, Alshiban AM, Alomar MA et a (2017) Knowledge of and Attitude Toward Repetitive Transcranial Magnetic Stimulation Among Psychiatrists in Saudi Arabia. The journal of ECT 33:30-35 